\title{
LA ATENUACIÓN EN LA JUSTIFICACIÓN ARGUMENTATIVA. UN ESTUDIO APLICADO AL DISCURSO DE INFORMANTES CON EDUCACIÓN UNIVERSITARIA
}

\author{
THE ATTENUATION DEVICE IN \\ ARGUMENTATIVE JUSTIFICATION. A \\ STUDY APPLIED TO THE DISCOURSE \\ OF BACHELOR DEGREE SPEAKERS
}


El contenido de este artículo forma parte de una investigación más amplia sobre la argumentación, entendida como macro-operación de la lógica natural mediante la cual se expone un punto de vista o un juicio a través de operaciones discursivas como la justificación. El objetivo principal es describir el uso de la atenuación en una muestra de justificaciones argumentativas emitidas por informantes con escolaridad mínima de licenciatura terminada.

Con un enfoque metodológico cualitativo: se dimensiona el valor retórico-lógico de la atenuación, definida como procedimiento discursivo para suavizar o mitigar los enunciados a través de estrategias relacionadas con el cuidado de la imagen; se distingue la atenuación cortés de la que se emplea como estrategia pragmática para conseguir algún otro fin; y se describe la incidencia de la cultura y la ideología en ese procedimiento utilizado para reducir los efectos negativos en la interpretación de lo dicho.

Además, mediante técnicas cuantitativas, se delimita la distribución sociolingüística del empleo de cada uno de los elementos analizados en su correlación con las variables de 'edad' y 'sexo'; y se identifican los indicadores de atenuación más frecuentes en la muestra examinada.

PALABRAS ClaVE: lógica natural, argumentación, justificación, atenuación

The research found in this article is part of a more extensive study about argumentation as a natural logic macro-operation through which the speaker states a point of view (or an opinion) by performing discursive procedures such as justification. Its main objective is to describe the uses of mitigation in argumentative justifications. The sample submitted to examination includes argumentative justifications uttered by individuals with college degree as minimal education level.

From a qualitative approach, this article:

States the rhetorical and logical value of 'attenuation', defined as a discursive procedure by which the emitter mitigates his/her utterances through some strategies related to face-work.

Distinguishes the uses of mitigation as a demonstration of courtesy, and as a pragmatic strategy to achieve some other pragmatic purpose; and Describes the impact of culture and ideology in the use of mitigation strategies.

Furthermore, quantitative techniques are applied: to delimit the sociolinguistic distribution of each of the elements analyzed, in correlation with 'age' and 'sex' variation; and also to identify the most common attenuation indicators found in the selected sample.

KEY WORDS: natural logic, argumentation, justification, attenuation

FECHA DE RECEPCIÓN: 24/06/2015

FECHA DE ACEPTACión: 7/09/2015 


\section{LA ATENUACIÓN EN LA JUSTIFICACIÓN ARGUMENTATIVA. UN ESTUDIO APLICADO AL DISCURSO DE INFORMANTES CON EDUCACIÓN UNIVERSITARIA}

\section{THE ATTENUATION DEVICE IN ARGUMENTATIVE JUSTIFICATION. A STUDY APPLIED TO THE DISCOURSE OF BACHELOR DEGREE SPEAKERS}

Lidia Rodríguez Alfano Universidad Autónoma de Nuevo León

\section{Introducción}

El Proyecto para el Estudio Sociolingüístico del Español de España y América (PRESEEA), que es dirigido por Francisco Moreno Fernández, de la Universidad de Alcalá de Henares, España, se encuentra adscrito a la Asociación de Lingüística y Filología de América Latina (ALFAL); y comprende equipos de investigación que se dieron a la tarea de reunir corpus de entrevistas con el fin de contar con los materiales necesarios para contrastar el uso del español en 40 ciudades de habla hispana. En la etapa actual, distintos equipos realizan estudios sobre temas lingüísticos y del discurso, en ocasiones coordinados. En la dimensión discursiva, el tema es la atenuación, ${ }^{1}$ cuyo estudio está siendo realizado

\footnotetext{
${ }^{1}$ Los estudios Atenuación_PRESEEA son coordinados por Ana María Cestero
} 
por integrantes de diversos equipos, entre ellos el denominado Monterrey-PRESEEA.

En el presente artículo se reportan los resultados del estudio de la atenuación en una muestra: conformada por todas las argumentaciones emitidas por los 36 informantes que completaron la licenciatura como grado mínimo de escolaridad; y que ha sido tomada del corpus "El habla de Monterrey-PRESEEA" constituido por los textos orales y escritos de 108 entrevistas grabadas y transliteradas electrónicamente ${ }^{2}$ entre 2006 y 2010.

Los objetivos generales son: describir el uso de la atenuación en las justificaciones argumentativas producidas en esas entrevistas; e identificar su distribución según las variables 'edad' y 'sexo'. La pertinencia de estos objetivos radica en que la justificación es una de las operaciones discursivas más importantes que se realizan en la presentación de evidencias dentro de las argumentaciones; $y$, con el fin de conseguir la adhesión del receptor, es muy frecuente que el emisor atenúe sus referencias para evadir así su responsabilidad respecto a lo hecho/dicho.

Esta investigación se apega al propósito central del PRESEEA: posibilitar estudios sobre la variación dialectal del español; y sigue las sugerencias incluidas en Cestero y Rodríguez Alfano (2014), donde se plantean "dos modalidades básicas de investigación desde las cuales puede abordarse

(Universidad de Alcalá de Henares, España) y Lidia Rodríguez Alfano (Universidad Autónoma de Nuevo León), la responsable, a la vez, de todos los estudios aplicados al corpus Monterrey-PRESEEA.

${ }^{2}$ El corpus completo, en textos de audio y escritos fue publicado en Rodríguez Alfano (2012a). 
el estudio" que comparten, entre otros criterios metodológicos: la necesidad de utilizar un enfoque cualitativo que sustente la codificación de los datos; su complementación con el análisis cuantitativo de los resultados obtenidos para facilitar su contraste por parte de investigadores de otro(s) equipos PRESEEA; y el uso de la estadística descriptiva para identificar la distribución sociolingüística del uso de atenuación. Además, se sugiere incluir una explicación de los datos considerando variables geográficas, sociales y/o situacionales, las diferencias culturales y la posible incidencia de la ideología en el discurso. La diferencia es que, en la Modalidad A, los estudios se aplican a todas las variables y variantes de la atenuación que se presenten en la codificación del corpus sometido a estudio. En cambio, en la Modalidad B no se pretende examinar en forma exhaustiva el uso de la atenuación, sino analizar en profundidad una o varias variables lingüísticas, pragmáticas o discursivas, y/o articular diversos enfoques teórico-metodológicos en su estudio.

Asimismo, a la presente investigación le antecede la ponencia expuesta en el Congreso ALED, Puebla 2013, donde se recogió la experiencia de los miembros del equipo de investigadores de Monterrey; y la publicación electrónica de Rodríguez Alfano (2015), que contiene una propuesta más actualizada para estudios en la Modalidad B de los estudios de Atenuación_PRESEEA.

Con todos estos antecedentes se anotan nuevas diferencias entre lo planteado en Cestero y Rodríguez Alfano (2014) y Rodríguez Alfano (2015) y la presente investigación, en la que (a) no se ha hecho el recorte en la muestra para considerar 30 minutos de grabación por cada entrevista, con el 
fin de facilitar los estudios contrastivos en PRESEEA; y (b) tampoco se ha tomado a los actos de habla como unidad de análisis.

Los planteamientos teórico-metodológicos parten del concepto de 'discurso' entendido como realización de la lengua en todo acto de enunciación, y en cuyo estudio se han de considerar distintas dimensiones que permitan un análisis más completo. A partir de esta convicción, se articulan diversas propuestas como las consideraciones sobre la lógica del discurso en sus diferencias con la lógica del razonamiento (Perelman y Olbrechts-Tyteca, 1989: 1); la escuela de Neuchâtel sobre la lógica natural; y la perspectiva pragmática que comprende una definición en sentido amplio de 'diálogo' que toma en cuenta la incidencia de múltiples contextos (Rodríguez Alfano, 2010b: 24-26).

Con estas concepciones básicas, se aprovechan aportaciones de los estudios de la cortesía y de la atenuación en español; y se toma en cuenta que, en la interacción comunicativa de las entrevistas, los participantes no se conciben como entes individuales. Aun cuando en cada turno se escuche solo la voz de uno de ellos, este se constituye en vocero de un grupo social, de una institución, de una postura ideológica o política... De este modo, el receptor puede percibir la multiplicidad de voces que producen la complejidad del sujeto emisor y la polifonía del discurso (Rodríguez Alfano, 2004: 171-176). Atendiendo a esta complejidad del discurso en la dimensión dialógica, ${ }^{3}$ además del contexto situacional, en

\footnotetext{
${ }^{3}$ El análisis del discurso desde esta dimensión se reporta en Fonte y Rodríguez Alfano (2010).
} 
la presente investigación se analiza la atenuación en su relación con la sociedad y la cultura, y con el poder y la ideología. ${ }^{4}$

\section{Fundamentos teórico-metodológicos}

El concepto más amplio en la presente investigación es el de discurso, concebido como una unidad de estudio mayor que la frase/oración que se define a partir de las propuesta de Benveniste (1971: 12-18) sobre el acto de enunciación; esto es, el acto mediante el cual el sistema abstracto de la lengua se convierte en discurso al tiempo en que un 'yo' se dirige a un 'tú' en una situación dada y con un propósito comunicativo concreto. Así entendido, el discurso se constituye en categoría compleja que denota una práctica comunicativa y, a la vez, el producto de esa práctica. Por lo tanto, se admite que es un conjunto de actividades simbólicas que un emisor pone en acto mediante el uso de códigos verbales y no verbales en una situación específica, y que su estudio comprende diversas dimensiones analíticas.

Al considerar que las entrevistas del corpus MonterreyPRESEEA fueron planeadas con el fin de obtener muestras cercanas al lenguaje coloquial (propósito compartido por otros corpus de este proyecto internacional), se ha centrado el interés en esta situación comunicativa. Se toma en cuenta que lo que ahí se realiza es un acto de enunciación cuyos

\footnotetext{
${ }^{4}$ En este estudio, la consideración de la incidencia de la ideología se apoya en aportaciones de Thompson (1987); Haidar y Rodríguez Alfano (1996); y Ducrot (1988b), que fueron retomadas en Rodríguez Alfano (2004: 381-442).
} 
participantes, el entrevistador y el informante, emplean el español semi-formal y semi-informal (distinto al que se emite en situaciones más formales, como una conferencia, por ejemplo) en una conversación cara a cara; $y$, por tanto, el enfoque de la perspectiva dialógica es prioritario.

Asimismo, dado que el objeto de estudio es la atenuación que se produce en las justificaciones argumentativas, en su análisis se aprovechan aportaciones de la Escuela de Neuchâtel, especialmente de Grize (1984), sobre la argumentación en lógica natural o espontánea que corresponde al discurso, y difiere de la lógica formal en varios puntos, entre los que se destacan los siguientes:

- Contrariamente a la separación tradicional que distingue la "doxa" de los argumentos que se considerarían válidos en la demostración de una verdad, con Perelman y Olbrechts-Tyteca (1989: 5), se sitúan en igual nivel la retórica y la lógica, ya que en la argumentación discursiva se presentan tanto procedimientos donde la persuasión tiene un lugar preponderante, como procedimientos racionales que se han considerado como propios de la lógica formal.

- Consecuencia de lo anterior es que los instrumentos de comprobación esenciales en la lógica clásica (implicación y sustitución -'si p es falso, q es verdadero', y viceversa) no son necesariamente válidos en la lógica espontánea, donde la valoración de un elemento como 'verdadero' no necesariamente conduce a excluir a los demás por considerarlos 'falsos': puede admitirse la paradoja de sustituir lo verdadero 
con algo más que también es verdadero y lo falso con algún otro elemento también falso.

- Es discurso de acción, lo que condiciona su naturaleza factual-deductiva y su carácter dialógico (aun cuando no estén presentes los interlocutores del emisor, por ejemplo en la argumentación periodística) con miras a conseguir una acción determinada, por lo que tiende a asegurar la aceptabilidad de lo que se plantea.

- Implica la esquematización del referente (de aquello de lo cual se habla) con base en preconstruidos que Grize clasifica como: situacionales, interdiscursivos e ideológicos. ${ }^{5}$

La lógica natural comprende la realización de cuatro macro-operaciones del discurso: demostración (de una verdad), descripción (topográfica, de objetos, o de procesos), narración, y argumentación. Esta última macrooperación discursiva se caracteriza por la presencia de un cierto grado de persuasión, e implica "una representación elaborada en lenguaje natural por un sujeto $\mathrm{A}$, con el propósito de volverla aceptable y verosímil para un sujeto $\mathrm{B}$ en una situación $S{ }^{36} \mathrm{Al}$ introducirla en su discurso, el hablante realiza la esquematización de sus referencias mientras expone una opinión o un juicio acerca de un objeto/persona o acontecimiento con el propósito de conseguir la adhesión

\footnotetext{
${ }^{5}$ Grize (1984: 194-195, 214-216, 222-223 y 226-227) citado por Rodríguez Alfano, 2004: 297-298.

${ }^{6}$ Traducción del francés de Lidia Rodríguez Alfano (2004: 295), en su referencia a Grize (1984).
} 
de su interlocutor, o bien de sostener su punto de vista, reafirmándolo.

En el presente estudio, los criterios seguidos para seleccionar un fragmento de discurso como 'argumentación' es que incluya la presentación de evidencias mediante la cual el hablante se propone dar mayor verosimilitud a lo enunciado. En esta fase de la argumentación se realizan diversas operaciones que son compartidas por las otras tres macro-operaciones de la lógica natural (como la del 'anclaje' a conocimientos compartidos por los interlocutores en un diálogo), y también aquellas que le son propias, entre las cuales se halla la justificación cuya función es asegurar la credibilidad de lo que se argumenta y que comprende diversos tipos de razonamiento: la deducción, que a su vez incluye el empleo de argumentos cuasilógicos; la inducción, que conduce a un principio general a partir de ejemplos; la analogía, y la explicación (Rodríguez Alfano, 2004: 321-322).

Para fines del presente estudio se articulan propuestas de Grize (1990: 104-109) y de Borel (1987) para plantear la distinción entre la operación argumentativa de la justificación y el razonamiento justificativo de la explicación, con base en Rodríguez Alfano (2013). Tanto la justificación como la explicación se utilizan para reforzar la validez de lo argumentado mediante la exposición de relaciones causales suceso $\rightarrow$ consecuencia que dan un mayor grado de certidumbre a los enunciados. La diferencia es que, en la explicación, simplemente se ofrece información adicional para aclarar las razones por las cuales ha dicho/hecho lo que refiere en sus argumentos, por lo que coincide en ciertos casos con las operaciones de enfoque o éclairage (aclaración). En cambio, 
la justificación es admitida como tal por el receptor solamente si contiene una prueba suficiente para exonerar de toda responsabilidad (acerca de lo dicho/hecho) a quien argumenta. ${ }^{7}$ En ocasiones estas dos operaciones argumentativas se traslapan, como se observa en los siguientes ejemplos:

(1) ... ya voy a México y ya me dicen oye trais un acento / norteño más / más grande que el de los norteños de allá / ya / ya se me quitó el manito y el sonsonete de allá ¿verdá? (HMP099).

(2) ...López Obrador lo que está haciendo es / este / sí / una inestabilidad / social muy grave / que nosotros no / no nos lo merecemos ¿veá? / claro que / que yo sé que / Calderón / no que tú digas ¡ah! Calderón es lo máximo / pero sí / era el me / es el mejor en estos momentos para / porque es pacífico (HMP033).

En (1), el informante explica las razones por las cuales le dicen que ya habla con acento más norteño que los nativos de acá: ya no dice "manito" ni tiene el "sonsonete de allá (de la capital); en cambio, en (2), justifica su adhesión a la postura política del partido de la derecha mediante la presentación de evidencias en el sentido de que Calderón (entonces líder de ese partido) era "el mejor" candidato a la presidencia en ese momento histórico; y, como contraargumento, un juicio negativo sobre el líder del partido de izquierda (López Obrador).

\footnotetext{
${ }^{7}$ Para la distinción entre la justificación y la explicación, se articulan las propuestas de Grize (1990: 104-109) y lo expuesto en el artículo de Borel (1987). Partes de esta distinción se hallan en Rodríguez Alfano (2004; 2012b; 2013).
} 


\section{La atenuación en la justificación argumentativa}

A diferencia de los procedimientos racionales de la lógica natural entre los que se ubica la explicación, en la justificación argumentativa también se utiliza otro tipo de procedimientos que no se relacionan tanto (como los anteriores) con los usados en la lógica formal, sino que cumplen funciones comunicativas de otra índole, y son marcados más fuertemente por la persuasión; pueden, por tanto, considerarse como 'procedimientos discursivos retórico-lógicos' al ser utilizados para conseguir la adhesión del interlocutor. Entre ellos se ubica la atenuación, que consiste en disminuir, suavizar o mitigar lo dicho. En su empleo puede complementarse u oponerse a la intensificación que, por el contrario, aumenta o pondera lo referido. Estos procedimientos retórico-lógicos no han sido examinados con precisión en los estudios de la argumentación en lógica natural, por lo que, para su descripción en este estudio, se articulan los planteamientos de la Escuela de Neuchâtel con propuestas que provienen de la pragmática lingüística.

Aunque sean opuestos en su significación, estos dos procedimientos discursivos tienen en común la función de servir para alcanzar un propósito comunicativo dado. Entre las estrategias retórico-lógicas en que se apoyan se encuentran: para la intensificación, el énfasis y la reiteración, que indican la seguridad en lo que se afirma; y para la atenuación, una serie de recursos para mitigar o suavizar lo argumentado.

En la identificación de indicadores de atenuación e intensificación, se ha considerado el hecho de que en la lengua 
española no se cuenta con elementos morfológicos o léxicos que marquen estos procedimientos discursivos en forma exclusiva. Muchos de los que, en la recepción por un hablante nativo, pudieran ser interpretados como indicadores de atenuación, en otro acto de enunciación podrían funcionar como marcadores de intensificación o de otras funciones pragmático-discursivas. Este es el caso de elementos paralingüísticos como la risa, la selección léxica de adverbios, adjetivos y aun de adversativas, etc. La identificación de la función atenuadora se apoya más bien en el co-texto (lo que antecede y sigue a su introducción en el discurso). Así, la acotación al ámbito personal que se evidencia en el uso de yo, mi, me..., y la consideración del interlocutor dada en el nosotros inclusivo, no siempre mitigan las referencias; estos elementos deícticos incluso llegan a marcar el discurso enfático, por ejemplo cuando se emplean en sentido irónico (Rodríguez Alfano, 2010a; 2012a).

Por lo tanto, en la presente investigación sobre usos de la atenuación, se atiende muy especialmente al funcionamiento de lo implícito (Rodríguez Alfano, 2002: 227-263) que incluye el presupuesto y el sobrentendido, según los define Ducrot (1986: 21-23); ${ }^{8}$ y se considera en forma relevante otra operación de la lógica natural, la modalización, que comprende procedimientos discursivos mediante los cuales el hablante acorta o aumenta la distancia entre sí mismo y sus enunciados, o bien incluye a su interlocutor en sus referencias, con el fin de evadir/asumir sus compromisos

${ }^{8}$ Para la consideración de lo implícito en el discurso, véase Rodríguez Alfano (2002; 2004). 
modales —alético, en relación con la verosimilitud; epistémico, relacionado con el grado de conocimiento que admite poseer acerca de lo que argumenta; y deóntico, aplicado al deber ser/hacer (Rodríguez Alfano, 2012b: 4). Así, en el siguiente ejemplo, la atenuación de lo argumentado revela un funcionamiento modal relacionado con el compromiso alético:

(3) ...pos stá interesante <énfasis > / a mí me encantó / como novela me encantó <énfasis> (...) (es) agilísima / te / quieres seguir leyendo / son capítulos muy cortos / en mi opinión bien / bien traducidos (...) y muy / muy ágil / muy interesantes / haz de cuenta que fuera un / un thriller ¿verdá? (HMP108).

En (3), la acotación a la perspectiva personal (a mí me..., en mi opinión personal...) y la emisión de enunciados en la función conativa que revela la inclusión del interlocutor (te, quieres, haz de cuenta) se consideran estrategias de modalización que disminuyen el compromiso alético que todo emisor establece respecto a la verosimilitud de lo que enuncia, en este caso, en sus argumentos. En consecuencia, constituyen estrategias retóricas de la atenuación, que se complementan con procedimientos de intensificación indicados por el uso del verbo 'encantar' que denota la acción de gustar en sumo grado; adjetivos ('ágil', 'interesante') que, sobre todo en el grado superlativo (con el sufijo 'ísimo', y los adverbios 'bien' y 'muy'), ponderan el valor del referente (una novela).

Asimismo, la atenuación se constituye en estrategia de modalización discursiva cuando la informante de la en- 
trevista HMP104 evade el cumplimiento del compromiso epistémico al tiempo que responde a la pregunta que se le hiciera sobre el origen de sus abuelos maternos:

(4) ...ellos vivían en esa / en ese<alargamiento/> / lugar / am<alargamiento/> / ¡ay! / "Los arro-yos<alargamiento>" / "Los arroyos" se llama el / ese lugar / no sé si era / como especie de rancho o hacienda eso sí nunca (lo) supe porque / no / no fuimos mucho par'allá porque como mi mamá siempre vivió en General Terán / era / nada más yo sabía que / ella / sus padres venían de allá pero no / no estaba segura / nunca / nunca fuimos o si acaso fui no recuerdo / porque he de haber estado muy pequeña (HMP104).

$\mathrm{Al}$ articular su respuesta, la entrevistada centra su argumentación en la presentación de evidencias que justifiquen su desconocimiento sobre la información que se le solicita, $\mathrm{y}$, por ende, en la evasión de su compromiso epistémico; y con este fin utiliza el procedimiento discursivo de la atenuación, que realiza mediante el empleo de estrategias retórico-lógicas que incluyen: (a) acotación de lo dicho al ámbito personal (evitando la generalización) y negación de un conocimiento preciso sobre aquello de lo cual se habla (no sé, eso sí nunca supe...); (b) uso de la vaguedad en las referencias (una especie de..., si acaso fui...); (c) una pronunciación marcada por el alargamiento de los sonidos mientras se planea el discurso subsiguiente, que indica inseguridad acerca de lo que se expone mediante la selección léxica; y la referencia explícita a esa inseguridad respecto a lo enunciado (nada más yo sabía que... pero no estaba segura). 
Por otra parte, en el empleo de intensificación y atenuación en las justificaciones argumentativas emitidas en el siguiente fragmento de otra de las entrevistas de la muestra, se revela el cuidado de la imagen (face care), cuyo funcionamiento pragmático fue descrito inicialmente por Goffman (1967: 5) y estudiado con mayor profundidad por Arundale (2006: 193-216):

(5) (E: Pero los alumnos / usted / ¿llegó a ver / cómo este / pos la diferencia / de unos años / atrás / a ahora / o a los últimos años / que trabajó? / pues los muchachos ya no son / ya no somos como antes) I: No ya / no / ya no (risas) (E: Los niños...) Yo / yo digo que teníamos que cambiar en algo ¿verdad? (...) Porque realmente / este / tener una disciplina tan férrea / no me arrepiento de nada / (risas) no me arrepiento de nada porque / tuvimos muchísimos / excelentes exal- / alumnos (...) Y este / y es más / nos encuentran en la calle nos ven con mucho gusto / nos saludan / nos dicen "maestra / como ustedes no hubo otras" / puras mentiras ¿verdá? (risas) (HMP107).

En la realización de procedimientos de intensificación en (5), subyace el cuidado de la imagen propia, que se manifiesta en la ponderación de un rasgo positivo con que se auto-representa la informante como 'buena maestra' (apego a la disciplina férrea de la que no me arrepiento); la cita expuesta en estilo directo de una valoración positiva emitida por sus exalumnos respecto a sus profesoras (como ustedes no hubo otras); además del uso de un adverbio que aumenta la verosimilitud de lo argumentado (realmente) y de un ad- 
jetivo (excelentes) unido al cuantificador (mucho) que aparece también en superlativo (muchísimos), recurso retórico que también apoya a la intensificación. Sin embargo, toda esa argumentación es suavizada mediante estrategias de atenuación: la repetición (en eco) de los términos usados por la entrevistadora al expresar un comentario (los alumnos ya no somos como antes) y el recurso modal de incluirse en esa referencia ( $y o$ digo que teníamos que cambiar en algo); la aparente anulación del halago expresado por sus exalumnos, y que ha citado en estilo directo ('puras mentiras'); el uso la pregunta de retroalimentación '¿verdad?/¿verdá?', mediante la cual incluye a su interlocutora en sus enunciados; y la continua introducción de la risa. Todas estas estrategias tienen un valor retórico en cuanto sirven a la informante para mitigar la ponderación de los valores que se ha adjudicado en su auto-representación y, en consecuencia, cuidar tanto su auto-imagen como la de su interlocutora y aun la de los emisores de la cita introducida en refuerzo de su argumentación.

Las actividades del cuidado de la imagen no siempre se relacionan con la cortesía, como lo ha propuesto Briz (2007: 2), quien distingue dos funciones de la atenuación como procedimiento discursivo cuyo empleo reduce los posibles efectos negativos en la interpretación de lo dicho: la atenuación cortés, que se emplea en apego a las normas compartidas por la comunidad de hablantes acerca de lo que se considera "buen comportamiento verbal" y que, en el español usado en Monterrey implica el cuidado de la imagen del interlocutor o de otro(s); y la atenuación pragmática que se constituye en recurso de la persuasión al encaminarse ha- 
cia el cuidado de la autoimagen para lograr algún propósito. Véanse los siguientes ejemplos:

(6) (E: <entre_risas> déjame agarrar aire y terminar el agua // porque sí // me preocupaba no haber llegado a tiempo) I: Ah nombre no te apures / nada qué ver / ya me imaginé dije / algo se le cuatrapeó porque no es / aparte no es muy fácil // la calle / como es una calle chiquita... (HMP068).

(7) ...te digo que soy media exigentilla / sí / pero fíjate que a mí me pasó una cosa con una nieta / se fueron / mi hijo / y su esposa salieron y me pidieron que si me iba a quedar a dormir con ellos / nada más a dormir / y tenían dos muchachas que les ayudaban /jah! / claro que sí / yo iba para que se acostaran / y que se levantaran / ir para colegio y todo / el niño que es el mayor / dijo ya me voy a acostar porque mañana me tengo que levantar ma- / muy temprano creo que eran como las nueve de la noche / ya lo acompañé / rezamos y todo / y luego bajé y les dije a las niñas / yo creo que apagamos la tele ¿verdad! / ¡no! / abuelita / ¡no! / que quién sabe qué / estaban viendo caricaturas / pero caricaturas / de las que ya tenían ahí en su casa (HMP108).

En (6), la atenuación es cortés, la informante atenúa la impuntualidad en cuidado de la imagen de su interlocutora; y, en (7), es estratégico-pragmática en cuanto, con el fin de salvaguardar su imagen, la informante atenúa la referencia del calificativo 'exigente' (rasgo que se adjudica en su autorepresentación) mediante el uso del diminutivo (exigentilla).

La jerarquización de las categorías teóricas consideradas en este estudio se ilustra en la siguiente figura: 
Figura 1. Orden jerárquico de las categorías consideradas en el estudio de la atenuación en la justificación argumentativa

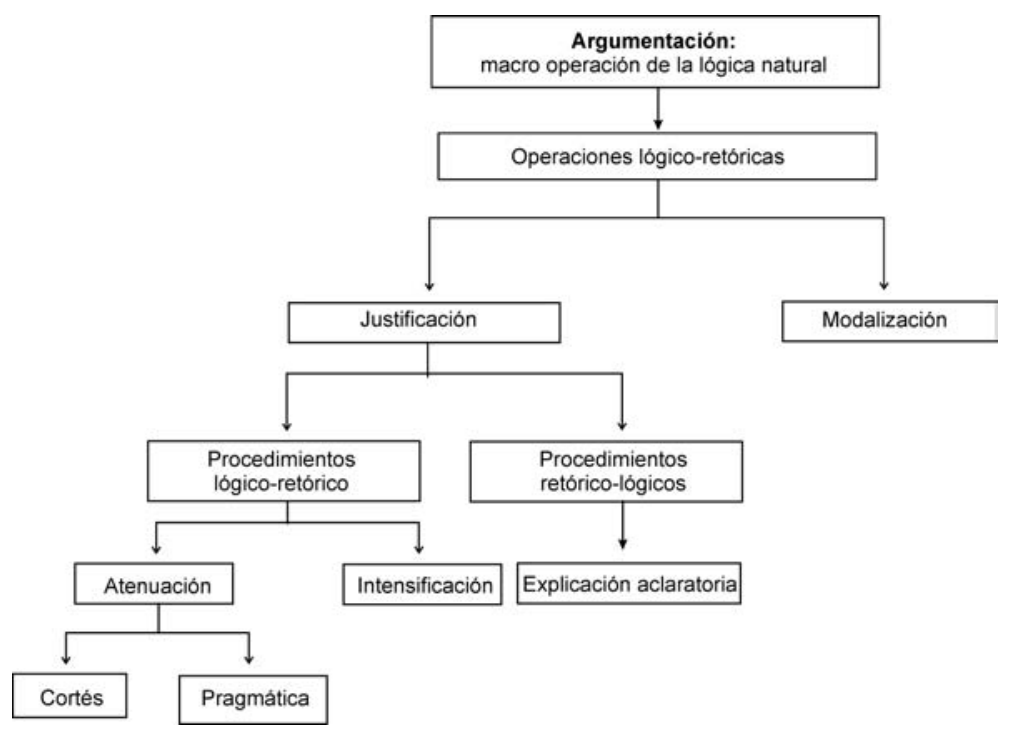

En esta figura se destacan (con caracteres y flechas de mayores dimensiones) las categorías que atañen más directamente a las funciones de la atenuación en la justificación argumentativa.

\section{Identificación de indicadores de atenuación}

Para la identificación de elementos que indican la presencia de la atenuación en un argumento, se ha aprovechado la lista de los que se reportan en Cestero y Albelda (2012); y, con esta base, se ha seguido el criterio que se ilustra mediante los ejemplos siguientes: 
(8) ...en mi caso particular y mi papá / pos él era de Morelia / se educó en Morelia / a principios del siglo pasado (...) allá eran / los papás mandaban y los hijos obedecían punto / ya aquí en Monterrey / era diferente porque yo lo veía con mis amigas (HMP108).

(9) (E: es maestra / ¿y el salario que ella tiene?) Ay ahí sí no / (E: no sabe) no (E: Okay) gana muy poquito ella la pobrecita (risas) / pero no / no / no sé / para qué te echo mentiras (HMP107).

En (8), la atenuación es indicada sólo por la selección léxica (eufemística) del calificativo "diferente" que disminuye el efecto que causaría el empleo de un calificativo ponderativo (como mejor) que comprometería a la informante como emisora de una crítica a una ciudad como Morelia en contraste con Monterrey (su ciudad natal); y en (9), la atenuación marcada en la selección léxica de poco (que disminuye la cantidad referida) y pobre (que minimiza el valor de quien labora como maestra) es reforzada por el modificador adverbial muy más la modificación interna causada por el empleo del diminutivo: poquito, pobrecita, y la risa.

\section{Atenuación como estrategia ideológica}

De acuerdo con el enfoque de la polifonía discursiva, el emisor, además de expresar ideas, deseos o sentimientos, participa activamente en la reconstrucción del sentido explícito e implícito, al manifestar en sus enunciados diversas voces, que conforman citas del discurso ya enunciado con anterioridad y que se reproduce en procesos de intertextualidad / 
interdiscursividad. ${ }^{9}$ Entre esas voces se hallan las que provienen de posturas institucionales e ideológicas, como se evidencia enseguida:

(10) ...y de casada como que ya tienes que compartir / e cosas y / tomar opiniones / vaya / poner a consideración / para que pueda prevalecer pues / una verdadera comunicación en pareja ¿verdad? (...E.....y las películas, ¿las ves dobladas / o subtituladas / o dobladas y subtituladas?) I: Bueno mi esposo siempre quiere dobladas / no le gusta mortificarse la existencia y como las veo con él / bueno / me aguanto (...) bueno no me aguanto me da igual (HMP071).

En la argumentación expuesta en este fragmento del diálogo, ante la pregunta de la entrevistadora que la informante habría de contestar con alusión a su preferencia personal, esta responde: mi esposo siempre quiere dobladas / no le gusta mortificarse la existencia y como las veo con él / bueno / me aguanto. Así se manifiesta la realización de (a) la operación argumentativa de justificación que incluye estrategias retóricas propias de la atenuación como empleo del deíctico de segunda persona, que involucra al interlocutor en la acción designada por un verbo que indica la modalidad deóntica (tienes que); la autocorrección (bueno, no me aguanto, me da igual); además de algunos indicadores de

\footnotetext{
9 El concepto de 'interdiscursividad' definido inicialmente por Bajtin (1981: 297-317) fue replanteado por Kristeva (1997) bajo la designación de 'intertextualidad'. Por su parte, Rodríguez Alfano (2004: 175) lo articuló con propuestas de Ducrot (1988a) y de Voloshinov (1976) al describir las dimensiones del sujeto emisor en la polifonía discursiva; y en este sentido se retoma para este estudio.
} 
inseguridad (como que...); y, simultáneamente, (b) procedimientos discursivos que, como los descritos por Reboul (1986: 35-43), ya no corresponden a la lógica natural, sino que constituyen parte de una práctica social incidida por la triple relación implicativa poder $\Leftrightarrow$ ideología $\Leftrightarrow$ discurso y revelan un funcionamiento ideológico en la admisión, por parte de los dos interlocutores de la entrevista, de un $\boldsymbol{t o p o s}^{10}$ compartido por los participantes y cuyo valor de verdad no se somete a cuestionamiento alguno. En este caso, el topos reiterado constantemente y admitido como verdad incuestionable proviene de la ideología machista y se refiere al supuesto papel de "sumisión" de la mujer casada, que le exige compartir y aguantarse a fin de favorecer una verdadera comunicación de la pareja.

\section{Distribución sociolingüística de las argumentaciones}

Siguiendo el criterio compartido por todos los equipos del PRESEEA, los 36 informantes que conforman el universo de estudio en la presente investigación han sido ubicados en el nivel 3 de educación que corresponde a aquellos que cuentan con la licenciatura terminada como grado mínimo de

\footnotetext{
10 El concepto de topoi (plural de topos) fue empleado originalmente por Aristóteles con la denotación de 'lugares/tópicos' que son de conocimiento común y tienen contenido retórico. Ducrot (1988b: 102-106) lo emplea para designar elementos cuyo valor retórico se ha fijado en el sistema de la lengua; y, para fines de la presente investigación, este concepto se acerca al de 'preconstruidos ideológicos' en la concepción de Grize (1984) referida en la nota 3 del presente artículo.
} 
escolaridad; y se les ha clasificado en tres subgrupos donde los primeros dígitos indican la edad y el segundo dígito (3) es indicador de la escolaridad: 1-3, de 20 a 34 años; 2-3, de 35 a 54; y 3-3, mayores de 54.

Enseguida se exponen los resultados del análisis cuantitativo, siempre en orden de frecuencia y en consideración de la correlación sociolingüística según las variables 'edad' y 'sexo', de cada una de las categorías ilustradas en la figura 1. Primeramente se ofrecen los que corresponden a la categoría más amplia, la macro-operación argumentativa, sobre la cual se reporta que el total de las argumentaciones examinadas es de 1,609, y su distribución sociolingüística se ilustra en las gráficas siguientes:

Gráfica 1. Argumentaciones en relación con la variable 'sexo'

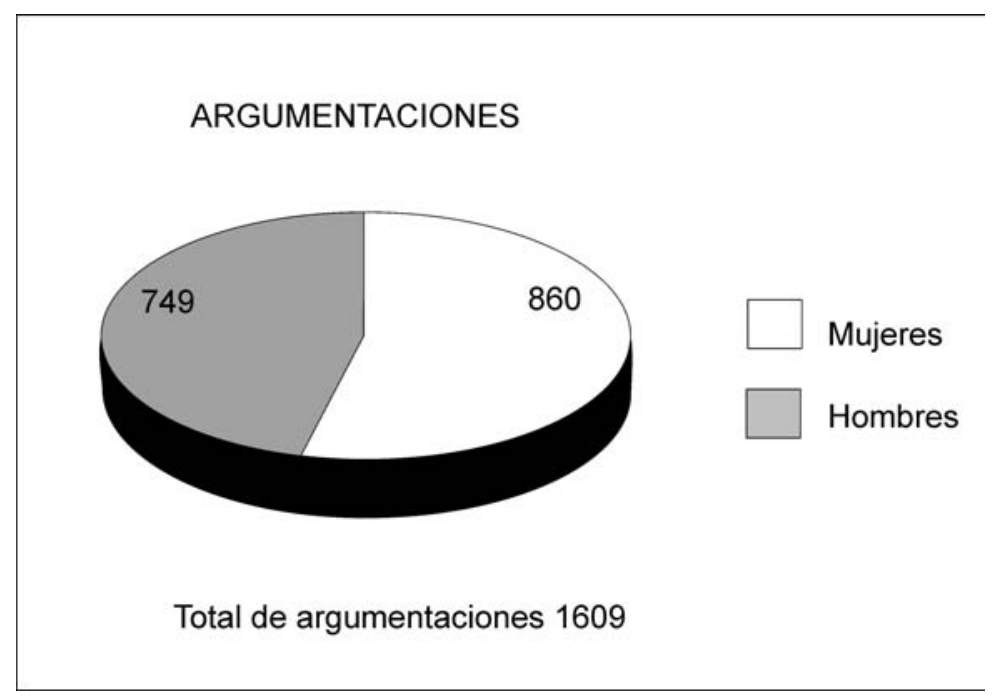


Gráfica 2. Argumentaciones en correlación 'sexo' y 'edad'

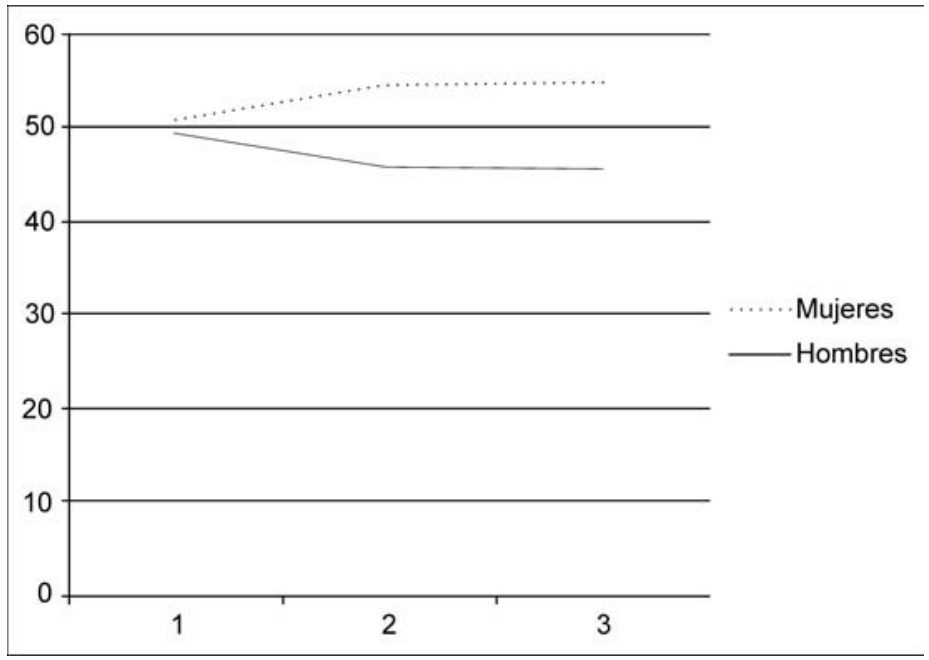

Como puede observarse en la gráfica 1, el grupo de las mujeres supera al de los hombres al exponer argumentos que incluyan la presentación de evidencias; y en la gráfica 2, donde se correlacionan las variables 'sexo' y 'edad', se muestra que cerca del $50 \%$ (esto es, 802 argumentaciones) corresponde al nivel más alto de edad (nivel 3-3, mayores de 54 años), y el resto, a los niveles 1-3 (entre 20 y 34 años) y 2-3 (entre 35 y 54), con 483 y 324 argumentaciones respectivamente. Esto es que, en la medida en que aumenta la edad, la diferencia en la cantidad de argumentaciones producidas por hombres y mujeres se hace más marcada. En el grupo de los jóvenes (nivel 1-3) la diferencia entre los sexos es mínima ( $0.72 \%$ a favor de las mujeres), mientas que en el nivel 2-3 aumenta en una proporción que casi se mantiene igual en el nivel 3-3. 
La categoría que sigue en el nivel jerárquico es la justificación relacionada con uno de sus procedimientos racionales considerados por los estudiosos de la lógica natural, la explicación. El resultado del análisis cuantitativo aplicado a la realización de justificaciones y explicaciones en co-relación con 'edad' y 'sexo' muestra que en todos los grupos hay más explicación que justificación; la mayor cantidad de justificaciones es producida por los hombres, quienes son seguidos por las mujeres del nivel 3-3; y son mujeres quienes entretejen explicaciones en sus justificaciones (excepto en el nivel 2-3, en el que también los hombres lo hacen en una pequeña proporción). En la siguiente gráfica se ilustran estos resultados:

\section{Gráfica 3. Operaciones}

de justificación/explicación

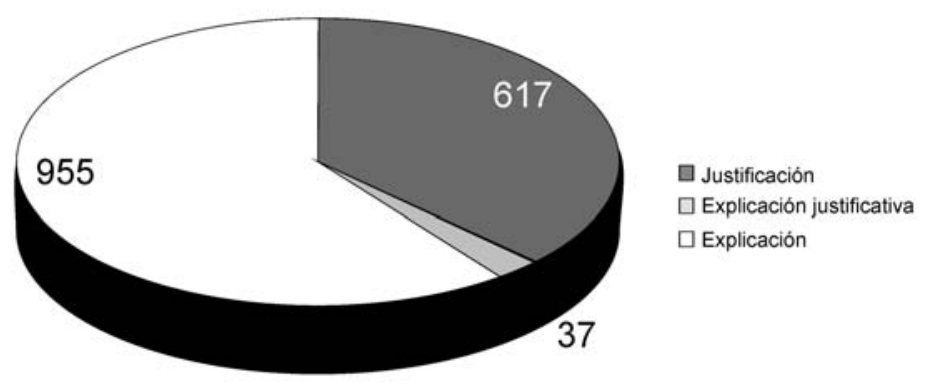

\section{Grupo 3 de educación Total de argumentaciones 1609}


Gráfica 4. Justificación/explicación en co-relación con 'edad' y 'sexo'

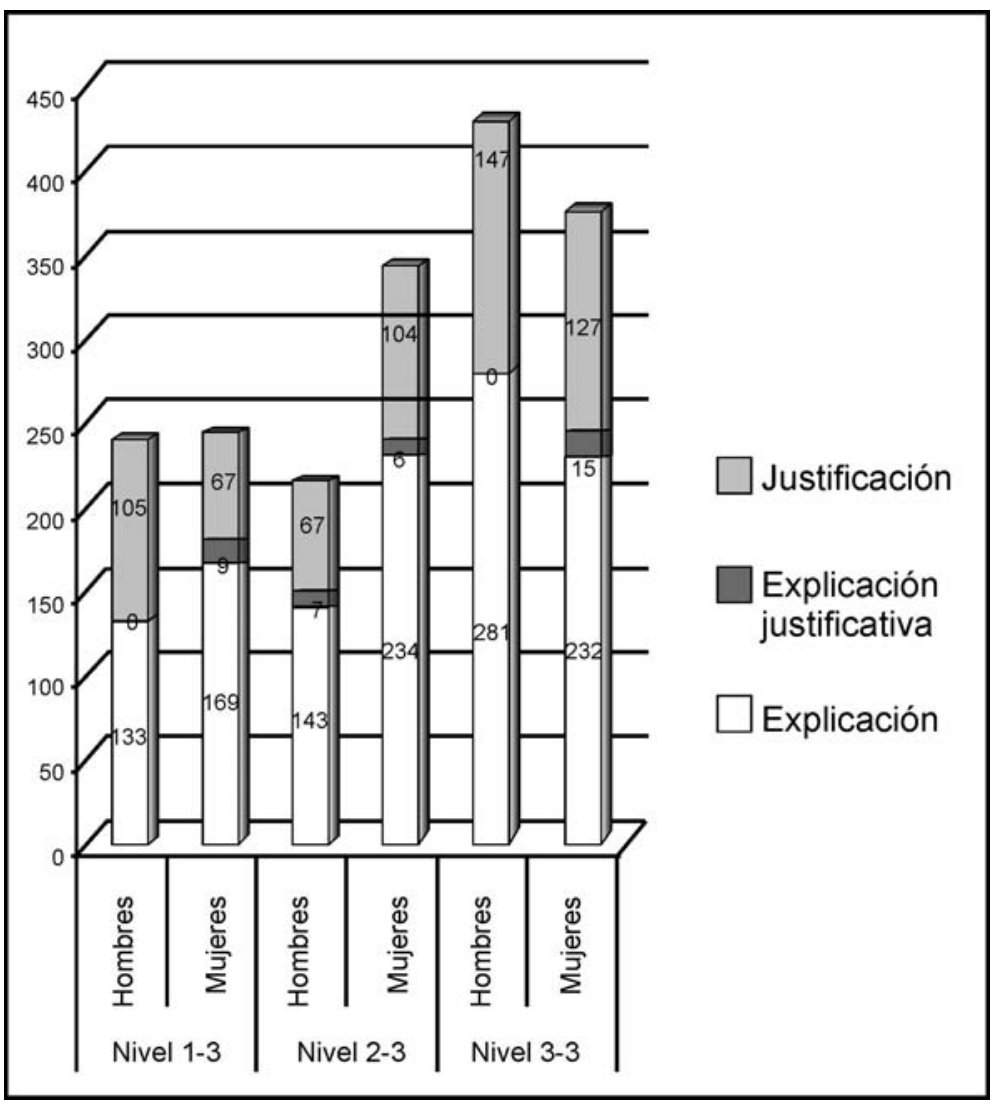

Enseguida se ofrecen los datos estadísticos referentes a la atenuación, donde se contrasta su empleo con el otro procedimiento discursivo argumentativo que se ha descrito, la intensificación. Su distribución sociolingüística se representa en la gráfica siguiente, en la que se observa que los argumentos atenuados superan a los intensificados: 
Gráfica 5. Atenuación e intensificación en justificaciones argumentativas, co-relación con 'sexo' y 'edad'

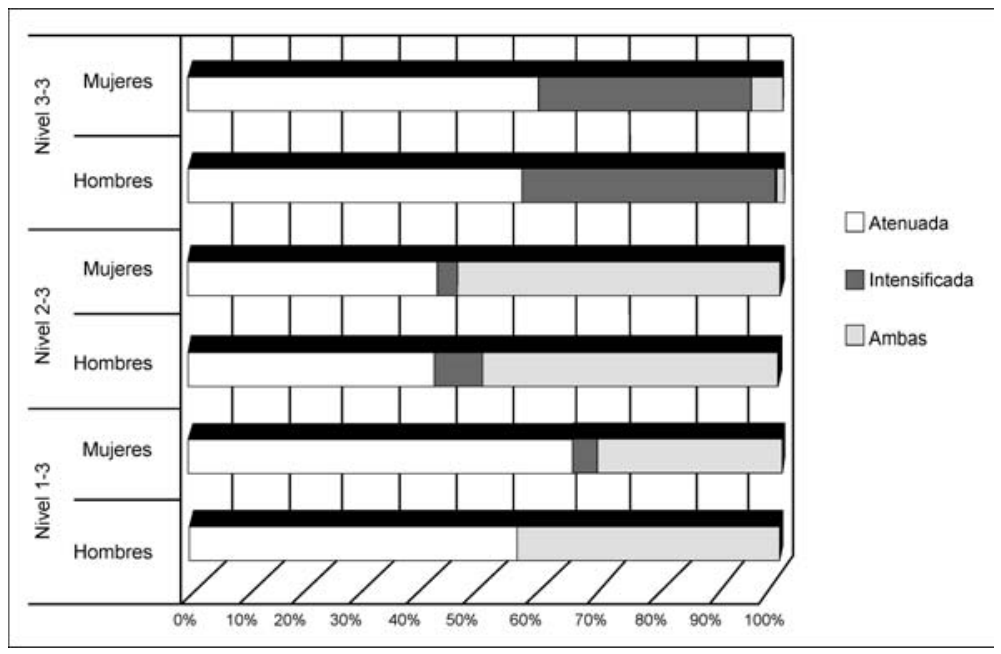

En la co-relación con las variables 'sexo' y 'edad', se encuentra que en el nivel 1-3 (de entre 20 y 34 años), el uso de la atenuación (sola o con explicaciones entretejidas) llega al $100 \%$ en el discurso de los hombres, mientras en un pequeño porcentaje de argumentos emitidos por mujeres se presenta la intensificación; en cambio, en el nivel 2-3 (de entre 35 y 54 años), son los hombres quienes intensifican sus argumentos en mayor proporción que las mujeres; y en el nivel 3-3 (mayores de 54), unos y otras intensifican, sin que por ello los argumentos intensificados superen el porcentaje de los atenuados.

Otro resultado del estudio es sobre la distribución sociolingüística del empleo de la atenuación en sus funciones cortés y pragmática: 
Gráfica 6. Uso de la atenuación cortés y estratégico-pragmática en justificaciones argumentativas

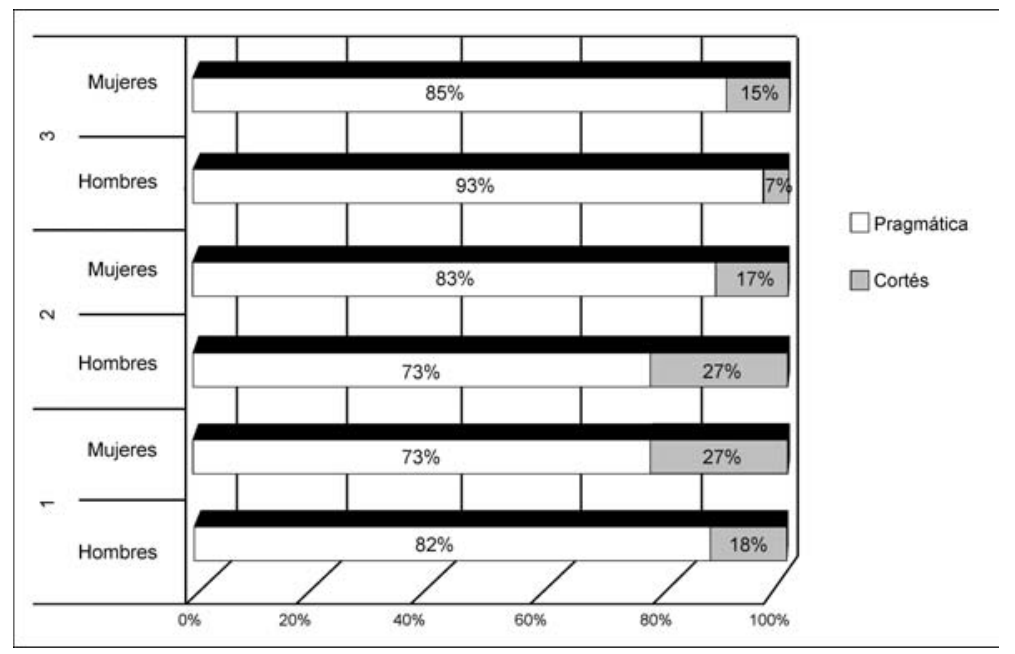

Como puede observarse, los usos de la atenuación pragmática, entendida como aquella mediante la cual el informante se propone alcanzar un fin (que puede ser en algún grado en provecho propio) son mucho más frecuentes, en comparación con el empleo de la atenuación cortés; y un dato de interés al respecto es que son los hombres del grupo 3 de edad quienes utilizan en menor proporción la atenuación cortés, esto es, sólo en un 7\% del total de justificaciones argumentativas; y los grupos que la utilizan con mayor frecuencia son los de las mujeres y los hombres "maduros" (entre 35 y 54 años de edad), en un 27\% del total.

Se concluye la exposición de resultados del análisis cuantitativo con la identificación de los indicadores de atenuación que tienen mayor frecuencia en su aparición en la 
muestra examinada, y para cuya designación y clasificación se aprovecharon los criterios expuestos en Cestero y Albelda (2012):

- Acotaciones, construcciones acotadoras de la opinión

- Apelativas, uso de formas de tratamiento (formas pronominales o nominales) y fórmulas apelativas convencionalizadas

- Control de contacto, partículas discursivas y expresiones de control de contacto

- Despersonalizaciones, impersonalizaciones

- Estructuras sintácticas condicionales, concesivas o temporales

- Excusas, partículas y construcciones con esta función

- Fingimiento, empleo de construcciones verbales y partículas discursivas que expresan fingimiento, incertidumbre, incompetencia o ignorancia

- Modal de duda, empleo de verbos, construcciones verbales, así como de partículas discursivas con valor modal que expresan opiniones en forma de duda o de probabilidad

- Modalizadores 'dislocados' de los tiempos verbales (uso del pospretérito y otros indicadores de lo no realizado)

- Modificadores externos, cuantificadores, minimizadores, aproximativos o difusores significativos

- Modificadores morfológicos internos

- Partículas discursivas

- Prosodia, empleo de elementos paralingüísticos o gestuales

- Reformulación, movimiento de reformulación

- Selección léxica, términos o expresiones más suaves en el contenido significativo y empleo de voces extranjeras. 
Su distribución sociolingüística es como sigue:

Gráfica 7. Indicadores de la atenuación en las justificaciones argumentativas

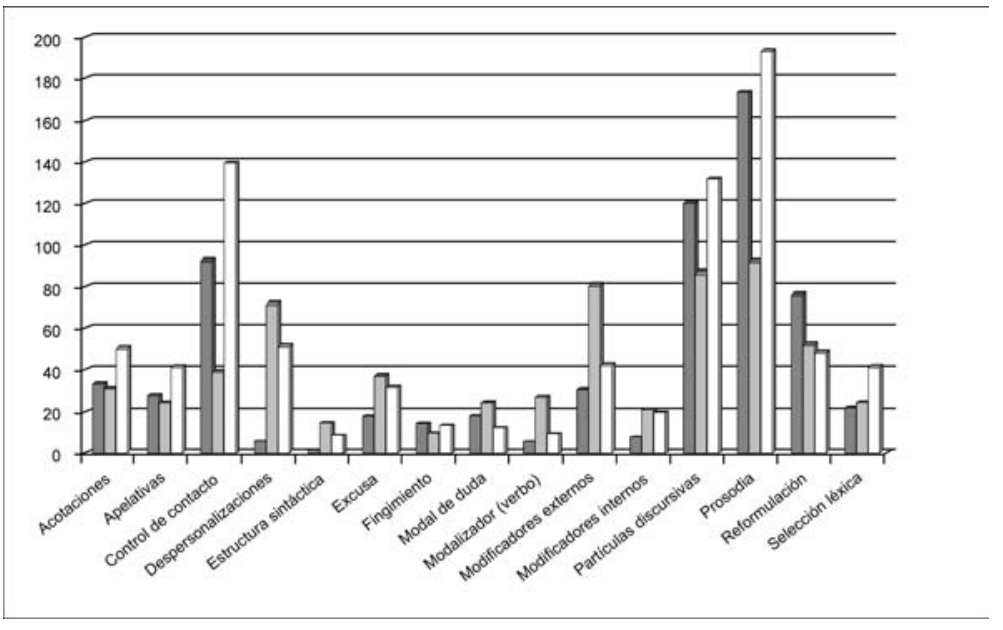

En esta gráfica resulta de interés observar que la más alta frecuencia recae en los indicadores prosódicos de la atenuación (entre los que se incluye el alargamiento de un sonido, que indica inseguridad en lo que se argumenta, y la entonación), seguidos por el control del contacto y el uso de partículas discursivas; $y$, en cuanto a su distribución sociolingüística -ilustrada mediante el uso de distintos colores para representar los niveles de edad 1, 2 y 3-, también es relevante notar que, en el discurso del grupo de edad 3, se emplean con mayor frecuencia que en los otros dos grupos, seis (de los quince) tipos de indicadores de atenuación aquí considerados; $y$, en contraste, en las justificaciones argumentativas emitidas por los más jóvenes (nivel 1-3), el em- 
pleo de algún tipo de indicador no llega a superar a los otros grupos (niveles 2-3 y 3-3).

\section{Consideraciones finales}

$1^{a}$ Mediante métodos del enfoque cualitativo, la definición de las categorías básicas se ha podido utilizar como herramienta para reconocer los presupuestos teórico-metodológicos en que se basa esta investigación; aclarar lo referente a la construcción y delimitación de la atenuación, entendida como objeto de estudio cuyo examen ha de ubicarse en la dimensión discursiva del análisis; y explicitar los criterios seguidos en la obtención, clasificación e interpretación de los datos. Otra aportación del enfoque cualitativo es que, además de la ilocutividad, se ha ubicado en otras dimensiones analíticas, entre ellas, la de los estudios del diálogo. El examen en profundidad desde la perspectiva dialógica muestra la relevancia de ciertos elementos del contexto situacional que modifican lo expuesto en una participación argumentativa que se co-construye en la medida en que los informantes reciben la retroalimentación de su interlocutor en el intercambio oral dado en las entrevistas sociolingüísticas (Koike, 2003: 11-24; Rodríguez Alfano, 2004: 295).

$2^{\text {a }}$ Los datos estadísticos muestran que en el total de argumentaciones emitidas por los informantes del nivel 3 de educación:

a) a medida que aumenta la edad de los informantes, la diferencia en la cantidad de argumentaciones producidas por hombres 
y mujeres se hace más marcada: en el nivel intermedio, la diferencia aumenta significativamente, y esa proporción casi se mantiene igual en el grupo de adultos mayores. Este dato puede explicarse en consideración de que, en el universo de estudio, la mayoría de las mujeres jóvenes, lo mismo que los hombres, son solteras(os), y tienen una ocupación fuera del hogar, lo cual les da oportunidad de conocer mejor los temas de conversación de la entrevista y, por tanto, mayor posibilidad de exponer argumentos en apoyo a sus puntos de vista, a diferencia de las ubicadas en los otros dos grupos de edad (donde hay una mayor proporción de madres/esposas);

b) hay un mayor uso de las explicaciones (emitidas con el fin de aclarar lo expuesto y así facilitar el proceso de interpretación por parte del receptor) que justificaciones (expuestas con el fin de evadir su responsabilidad respecto a lo dicho). Este dato manifiesta el principio de cooperación propuesto por Grice (1989: 22-40), y que subyace en la interacción dialógica realizada en las entrevistas de la muestra;

c) en la relación con la variable "sexo", se observa que los hombres utilizan más justificaciones que los deslinden de la responsabilidad de lo dicho, mientras las mujeres introducen más explicaciones dentro de sus justificaciones. Este dato prueba que, en este grupo social (de alto grado de escolaridad), las mujeres son más cooperativas y muestran una mayor inseguridad en su discurso (explican más para asegurarse de añadir verosimilitud a sus referencias) como lo ha reportado Deborah Tannen en sus múltiples estudios sobre la relación lenguaje-género;

d) tanto en el discurso de hombres y mujeres de todos los rangos de edad, la atenuación cumple más bien fines relacionados con 
el proyecto comunicativo del hablante, que con normas de la cortesía; y

e) los indicadores de la atenuación empleados con mayor frecuencia han sido los que se apoyan en la prosodia (entonación), el empleo de partículas discursivas y el control del contacto (preguntas de retroalimentación y otros tipos de apelación).

$3^{\text {a }}$ El propósito de la presente investigación ha sido contribuir en el perfeccionamiento de la metodología para el estudio de la atenuación, ya que en futuras investigaciones los resultados que se reportan han de contrastarse con los que se obtengan del análisis de la justificaciones atenuadas que introducen en su discurso los informantes de los demás corpus PRESEEA, especialmente los del nivel 3 de escolaridad; y

$4^{\text {a }}$ La metodología seguida en este estudio podrá utilizarse para el examen de la argumentación, la justificación y la atenuación, aplicado a cualquier otra muestra del lenguaje oral.

\section{Bibliografía}

Arundale, Robert (2006), "Face as relational and interactional: A communication framework for research on face, facework, and politeness", Journal of Politeness Research, 2, pp. 193-216.

Bajtin, Michael (1981), Estética de la creación verbal, traducción de Tatiana Bubnova, México, Siglo XXI.

Benveniste, Émile (1971), Problemas de lingüística general. Tomo I, traducción de Juan Almela, México, Siglo XXI. 
Borel, JeAn MARIE (1987), "Algunos aspectos del problema de la explicación”, Semiosis, 18, pp. 15-34.

Briz Gómez, Antonio (2007), "Para un análisis semántico, pragmático y sociopragmático de la cortesía atenuadora en España y América”, Lingüística Española Actual, 29, I, pp. 5-44.

Cestero, Ana María y Marta Albelda (2012). "La atenuación lingüística como fenómeno variable”, Oralia, 15, 77-124.

y Rodríguez Alfano, Lidia (2014), "Guía de Estudios de la atenuación en corpus PRESEEA", en http:// preseea.linguas.net/Metodología.aspx (publicado el 12 de junio de 2014).

Ducrot, Oswald (1986). El decir y lo dicho, traducción de Irene Agoff, Barcelona, Paidós.

- (1988a), Polifonía y argumentación. Conferencias del seminario Teoría de la Argumentación y Análisis del discurso, Calli, Universidad del Valle.

— (1988b), "Argumentación y 'topoi' argumentativos", Lenguaje en Contexto, I, 1 y 2, pp. 63-84.

Fonte Zarabozo, Irene y Lidia Rodríguez Alfano (2010), “Introducción”, en Irene Fonte Zarabozo y Lidia Rodríguez Alfaro (coords.), Perspectivas dialógicas en estudios del lenguaje, México, Universidad Autónoma de Nuevo León, pp. 7-18.

GofFMAn, ERving (1967), "On face-work. An analysis of ritual elements in social interaction" Interaction ritual. Essays on face-to-face behavior, Nueva York, Pantheon Books.

Grice, H. P. (1989), Studies in the Way of Words, Harvard, Harvard University Press. 
Grize, JeAn-Blaise (1984), De la logique á l'argumentation, Ginebra, Libraire Droz.

_ (1990), "La construction du discours: un point de vue sémiotique", en Michael Charolles, Sophie Fisher y Jacques Jayez (comps.), Le discours: Représentations et Interprétations, Nancy, Presses Universitaries de Nancy, pp. 11-18.

Haidar, Julieta y Lidia Rodríguez Alfano (1996), “Funcionamientos del poder y de la ideología en las practicas discursivas", Dimensión Antropológica, 3, 7, pp. 73-111. Koike, Dale April (2003), "La co-construcción del significado en español: elementos pragmáticos de la interacción dialógica”, en Dale April Koike (ed.), La coconstrucción del español de las Américas. Acercamientos discursivos, Toronto, Legas, pp. 11-24.

Kristeva, Julia (1997), "Bajtin, la palabra, el diálogo y la novela", en Intertextualité. Francia en el origen de un término y el desarrollo de un concepto, selección y traducción de Desiderio Navarro, La Habana, Casa de las Américas.

Perelman, Chaїm y Lucie Olbrechts-Tyteca (1989), La nueva retórica. Tratado de la argumentación, traducción de Julia Sevilla Muñoz, Madrid, Gredos.

Reboul, Olivier (1986), Lenguaje e ideología, México, Fondo de Cultura Económica.

Rodríguez Alfano, Lidia (2002), "Retórica y argumentación: lo implícito y las escalas adjetivo-argumentativas”, en Helena Beristáin (comp.), El abismo del lenguaje, México, Universidad Nacional Autónoma de México, pp. 227-263, Colección Bitácora de Retórica, 16. 
Rodríguez Alfano, Lidia (2004), Polifonía en la argumentación. Perspectiva interdisciplinaria. Los múltiples sentidos de un discurso sin fin, México, Instituto Nacional de Antropología e Historia, Universidad Nacional Autónoma de México, Universidad Autónoma de Nuevo León, Conarte.

(2010a), "La representación en el 'nosotros' de un sujeto colectivo", en Lidia Rodríguez Alfano (ed.), Procesos discursivos y funcionamientos del sentido. Representaciones, operaciones de la lógica natural, diálogo, y relaciones poder $\leftrightarrow$ ideología $\leftrightarrow$ discurso, Monterrey, Universidad Autónoma de Nuevo León, pp. 37-63.

(2010b), "A continuum of approaches to dialogue", en Dale Koike y Lidia Rodríguez Alfano (eds.), Dialogue in spanish: studies in functions and contexts, Ámsterdam, John Benjamin, pp. 1-27.

(2012a), Corpus Monterrey-PRESEEA, Monterrey, Universidad Autónoma de Nuevo León, Conacyt y Felina.

(2012b), "Indicadores de la modalización en El habla de Monterrey. De la elocutividad a la locutividad", en Lidia Rodríguez Alfano y María Eugenia Flores Treviño (eds.), Estudios lingüístico-discursivos en distintas emisiones lingüísticas reales. Aplicaciones concretas al corpus de El habla de Monterrey, Monterrey, Universidad Autónoma de Nuevo León, pp. 53-91.

(2013), "La atenuación en operaciones argumentativas de justificación en El habla de Monterrey-PRESEEA", ponencia presentada en el X Congreso Internacional de la Asociación Latinoamericana de Estudios del Dis- 
curso, Puebla, Benemérita Universidad Autónoma de Puebla, 28-31 de octubre.

Rodríguez Alfano, Lidia (2015), "La atenuación en un corpus sociolingüístico", Lenguas en Contexto, 12, pp. 167-172.

Thompson, John B. (1987), "Lenguaje e ideología", Zona abierta, 41-42, pp. 159-182.

Voloshinov, Valentín N. (1976), El signo ideológico y la filosofía del lenguaje, traducción de Rosa María Rússovich, Buenos Aires, Nueva Visión. 
